\title{
GAD Antibody-Associated Late-Onset Cerebellar Ataxia in Two Female Siblings
}

\author{
Joseph Kuchling ${ }^{a, c}$ Julia Shababi-Klein ${ }^{b}$ Astrid Nümann ${ }^{a}$ \\ Lea M. Gerischer ${ }^{a}$ Lutz Harms ${ }^{a, d, e}$ Harald Prüss $^{a, e, f}$ \\ Departments of ${ }^{a}$ Neurology and ${ }^{b}$ Psychiatry and Psychotherapy, ${ }^{b}$ NeuroCure Clinical \\ Research Center, ${ }^{d}$ Clinical and Experimental Multiple Sclerosis Research Center, and \\ ${ }^{e}$ Center for Autoimmune Encephalitis and Paraneoplastic Neurological Syndromes, \\ Charité - Universitätsmedizin Berlin, and ${ }^{\mathrm{f}}$ German Center for Neurodegenerative Diseases \\ (DZNE) Berlin, Berlin, Germany
}

\section{Key Words}

Autoantibodies · Autoimmunity $\cdot$ GAD $\cdot$ Anti-GAD65 $\cdot$ Anti-GAD antibody $\cdot$ Cerebellar ataxia

\begin{abstract}
Background: Anti-glutamic acid decarboxylase antibody (GAD-ab)-associated cerebellar ataxia is a rare neurological disorder characterized by cerebellar symptoms concomitant with high GAD-ab levels in serum and cerebrospinal fluid (CSF). Case Report: We report on 2 female siblings (aged 74 and 76 years) presenting with gradual progression of rotational vertigo, gait ataxia and vertical diplopia, continuously progressing for 6 months and 6 years, respectively. Autoimmune laboratory examinations showed remarkably increased serum and CSF GAD-ab levels. Their medical histories revealed late-onset type 1 diabetes mellitus (T1DM) and other concomitant autoimmune disorders (Grave's disease, Hashimoto's thyroiditis). Cerebral MRI and laboratory examinations were unremarkable. The diagnosis of GAD-ab-associated cerebellar ataxia with particular brainstem involvement was established in both women. After the exclusion of an underlying malignancy, immunosuppressive therapy has been initiated in both patients, which resulted in stabilization in one and in clinical improvement in the other patient. Discussion: The unique association of autoantibody-mediated cerebellar ataxia and late-onset T1DM in 2 siblings with similar clinical and paraclinical phenotypes strengthens the concept that hereditary factors might play a relevant role also in autoimmune diseases so far considered to be sporadic. Moreover, the occurrence of continuous vertical diplopia broadens the clinical spectrum of GAD-ab-associated neurological syndromes.


Kuchling et al.: GAD Antibody-Associated Late-Onset Cerebellar Ataxia in Two Female Siblings

\section{Background}

Anti-glutamic acid decarboxylase antibody (GAD-ab)-associated cerebellar ataxia is a rare, but increasingly detected, autoimmune neurological disorder characterized by the clinical presence of a cerebellar syndrome concomitant with high GAD-ab levels in serum and cerebrospinal fluid (CSF) [1-3].

Recent studies have linked GAD-ab cerebellar ataxia with type 1 diabetes mellitus (T1DM), other autoimmune endocrine disorders and, occasionally, even with paraneoplastic etiologies [4]. Although a positive family history of other autoimmune diseases such as T1DM and thyroiditis is common in GAD-ab cerebellar patients, previous studies did not determine a familial predisposition of GAD-ab cerebellar ataxia itself, which is thus considered to be sporadic [4].

The here described occurrence of GAD-ab cerebellar ataxia in 2 female siblings suggests that an underlying immunogenetical mechanism might additionally account for disease incidence and individual susceptibility.

\section{Case Presentation}

A 74-year-old Caucasian woman (patient 1) with no significant medical history presented with constant rotational vertigo, progressive gait ataxia with a tendency to fall to the right side and vertical diplopia increasing in right gaze. All symptoms were characterized by subacute onset with moderate progression over 6 months. Her elder sister, a 76-year-old Caucasian woman (patient 2) likewise presented with a 6-year-history of rotational vertigo, continuous gait ataxia and marked vertical diplopia. Symptoms were reported to have presented subacutely at onset and had initially been misdiagnosed as brainstem infarction.

In both patients, neurological examination revealed remarkably similar symptoms including gaze-evoked nystagmus and a slight abduction deficit of the right eye as well as ataxia and dysmetria in the upper and lower extremities with right-sided predominance. Because of the pronounced gait ataxia of both patients, they depended on a wheeled walker. Upon motor, reflex and sensory examination, no relevant findings were elicited, in particular no signs of dysarthria, peripheral neuropathy, spasticity, areflexia, vegetative symptoms or fasciculations that could have pointed to one of the hereditary ataxias, such as SCA1, 2, 3 and 6. Neuropsychological assessment did not reveal any substantial cognitive or memory deficits. Cerebral MRI findings showed mild generalized atrophy and multiple white matter lesions in both patients (fig. 1a-d). Except for glycated hemoglobin (HbA1c) levels, which were expectably elevated due to the existing T1DM, all other routine laboratory examinations were within normal limits. Comprehensive workup with extended autoimmune laboratory examinations revealed remarkably high serum and CSF GAD-ab levels in both siblings (fig. 1e, f). Other autoantibodies were negative, in particular antibodies against the NMDA, AMPA or GABA(B) receptor, LGI1, Caspr2, MAG, glycin receptor, or onconeuronal antibodies. Further CSF analysis showed slight pleocytosis in patient 1 and oligoclonal immunoglobulin bands in both patients.

In addition, the medical history of patient 1 revealed Hashimoto's thyroiditis with elevated serum antithyroid peroxidase antibody levels and recently diagnosed T1DM, whereas patient 2 exhibited a 7-year-history of Grave's disease with high levels of thyroid-stimulating immunoglobulins and a 6-year-history of insulin-dependent T1DM (table 1). Their family history revealed a 73-year-old brother suffering from rheumatism and another 75-year-old brother without relevant diseases. 
Kuchling et al.: GAD Antibody-Associated Late-Onset Cerebellar Ataxia in Two Female Siblings

The diagnosis of cerebellar ataxia with GAD-abs was established in both siblings based on the subacute onset, CSF inflammation in patient 1 , the very high GAD-ab titers, the partial response to immunotherapy, the age at onset and the typical constellation of insulindependent diabetes. Because of these findings, no genetic testing was performed. Since the detection of GAD-abs can occasionally indicate a paraneoplastic etiology [2,5], we undertook full-body computed tomography scans in both patients as well as whole-body and brain positron emission tomography in patient 1 , all of which showed no pathological findings, suggesting the absence of any malignancy.

Plasma separation by immunoadsorption was used to eliminate pathogenic antibodies from the serum compartment by binding to the extracorporeal columns. For this, patient 1 received 5 cycles every other day which led to subtle improvement of gait ataxia. Extended treatment with a further 10 cycles of immunoadsorption eventually resulted in remarkable improvement of gait which allowed the patient to walk without her walking frame. In patient 2, 7 cycles of plasmapheresis were administered every other day and resulted in subjective improvement of gait disability, and a slightly diminished degree of gaze-evoked nystagmus was seen on follow-up examination. However, relevant impairment persisted so that immunosuppressive therapy was escalated in both women to $1,000 \mathrm{mg}$ rituximab intravenously according to common practice [3]. Both patients gave their written informed consent for data analysis and the publication of this case report.

\section{Discussion}

In this familial case report, we present the occurrence of autoimmune GAD-abassociated cerebellar ataxia in 2 female siblings with resembling clinical phenotype concomitant with T1DM starting at a comparatively high age.

The pattern of GAD-ab-related autoimmune central nervous system (CNS) disorders is characterized by heterogeneous and occasionally overlapping clinical syndromes, including the Stiff Person syndrome, cerebellar ataxia, progressive encephalomyelitis with rigidity and myoclonus, limbic encephalitis, epilepsy and paraneoplastic syndromes $[2,5,6]$.

These CNS disorders often occur in association with other autoimmune diseases, mainly T1DM. Although the presence of GAD-abs is equally characteristic for both, >100-fold higher levels of GAD-abs in serum are frequently seen in neurological disorders compared to T1DM [2]. Moreover, intrathecal synthesis [7] and a different epitope specificity of GAD-abs [4] have been reported in neurological syndromes.

Whereas the immunogenetical relationship of T1DM with specific human leukocyte antigen genes is widely known and well established in the literature [8], data on immunogenetical features of GAD-ab-associated neurological disorders are scarce, likely due to the comparatively low incidence and the lack of reports on familial cases [4]. A markedly higher frequency of GAD-ab-seropositive cases was found in first-degree relatives of T1DM patients compared to healthy controls $[9,10]$. However, decisive inferences concerning a possible familial predisposition of GAD-ab neurological disorders cannot be drawn, since the causative mechanisms of requisitely high serum and CSF GAD-ab levels still remain unclear.

In our reported cases, the clinical and paraclinical presentations of both female siblings were remarkably similar. Both women exhibited typical cerebellar symptoms, i.e. continuous rotational vertigo, progressive gait ataxia and gaze-evoked nystagmus with subacute onset, detection of CSF oligoclonal bands and concomitant autoimmune diseases, implicating the diagnosis of GAD-ab cerebellar ataxia [1, 4]. 
The additional occurrence of persistent vertical diplopia, as described in both cases, is an uncommon finding. Although versatile symptoms including multifocal brainstem involvement have been reported in some patients [11], to our knowledge, the literature provides only 1 specific case of GAD-ab cerebellar ataxia with additional vertical diplopia, in which double vision lasted for only 10 days as an early prodromal symptom which had already disappeared before the eventual development of the full cerebellar syndrome [3]. In our cases, constant vertical diplopia was present at symptom onset and has been persisting throughout the entire disease course, implicating that diplopia can be added to the clinical features of GAD-ab cerebellar ataxia, broadening the known clinical spectrum $[1,3,4,11]$.

Interestingly, both related patients exhibited an identical phenotype even with regard to age at first symptoms, type of onset and CSF oligoclonal bands instead of developing 2 different neurological syndromes within the broad GAD-ab-associated spectrum. This might be related to the excessively high titers of GAD-abs that have been particularly linked to cerebellar ataxia in contrast to the Stiff Person syndrome $[1,6]$. The fact that high titers are required for clear CNS symptoms underscores the pathogenic role of GAD-abs in cerebellar damage, potentially due to enzyme inhibition of intracytoplasmic GAD or antibody-induced suppression of GABA release [1, 7, 12]. The clinical improvement in patient 1 further supports this concept, even though a definite causal relationship between GAD-ab autoimmunity and ataxia cannot be provided. This limitation is known in the field and has been attributed mainly to the presence of additional mechanisms, such as further antibodies against surface antigens [13] or pathogenic cytotoxic T cells [14].

The finding of GAD-abs in the 2 siblings suggests a hereditary component of GAD-ab cerebellar ataxia. Alternatively, the occurrence might be mere coincidence. However, this is rather unlikely as GAD-ab cerebellar ataxia is considered a rare disease that occurs only sporadically. Therefore, our case presentation suggests immunogenetical mechanisms possibly leading to a familial predisposition to develop autoimmune GAD-ab-associated neurological disorders.

Against the background of the diagnostic challenge, especially regarding the group of late-onset cerebellar ataxias [15], our case presentation gives rise to the question as to whether a positive family history for the disease allows the ruling in or ruling out of a hereditary, sporadic or autoimmune disorder in the respective individual diagnosis.

Taken together, by describing the unique association of late-onset autoantibodymediated ataxia and diabetes in 2 siblings, the cases strengthen the concept that hereditary factors might play a relevant role also in diseases thought to be primarily sporadic. Moreover, the occurrence of multifocal cerebellar and brainstem symptoms in both patients broadens the established clinical spectrum of GAD-ab-associated neurological syndromes. Nevertheless, further research is necessary to eventually understand the full scope of autoimmunity in GAD-ab-associated disorders, their relationship to other autoimmune diseases and their respective immuno- and pathogenetic mechanisms.

\section{Disclosure Statement}

The authors have nothing to disclose. 


\begin{tabular}{l|l}
\hline Case Rep Neurol 2014;6:264-270 \\
\hline DOI: $10.1159 / 000369784$ & $\begin{array}{l}\text { ○ 2014 S. Karger AG, Basel } \\
\text { www.karger.com/crn }\end{array}$ \\
\hline
\end{tabular}

Kuchling et al.: GAD Antibody-Associated Late-Onset Cerebellar Ataxia in Two Female Siblings

\section{References}

1 Saiz A, Blanco Y, Sabater L, González F, Bataller L, Casamitjana R, Ramió-Torrenta L, Graus F: Spectrum of neurological syndromes associated with glutamic acid decarboxylase antibodies: diagnostic clues for this association. Brain 2008;131:2553-2563.

2 Graus F, Saiz A, Dalmau J: Antibodies and neuronal autoimmune disorders of the CNS. J Neurol 2010;257:509-517.

-3 Ariño H, Gresa-Arribas N, Blanco Y, Martínez-Hernández E, Sabater L, Petit-Pedrol M, Rouco I, Bataller L, Dalmau J, Saiz A, Graus F: Cerebellar ataxia and glutamic acid decarboxylase antibodies: immunologic profile and long-term effect of immunotherapy. JAMA Neurol 2014;71:1009-1016.

-4 Honnorat J, Saiz A, Giometto B, Vincent A, Brieva L, Andres C, Maestre J, Fabien N, Vighetto A, Casamitjana R, Thivolet C, Tavolato B, Antoine JC, Trouillas P Graus F: Cerebellar ataxia with anti-glutamic acid decarboxylase antibodies: study of 14 patients. Arch Neurol 2001;58:225-230.

5 Leypoldt F, Wandinger K-P: Paraneoplastic neurological syndromes: paraneoplastic neurological syndromes. Clin Exp Immunol 2014;175:336-348.

-6 Dayalu P, Teener J: Stiff Person syndrome and other anti-GAD-associated neurologic disorders. Semin Neurol 2013;32:544-549.

7 Verrotti A, Scaparrotta A, Olivieri C, Chiarelli F: Seizures and type 1 diabetes mellitus: current state of knowledge. Eur J Endocrinol 2012;167:749-758.

8 Kulmala P, Savola K, Reijonen H, Veijola R, Vähäsalo P, Karjalainen J, Tuomilehto-Wolf E, Ilonen J, Tuomilehto J, Akerblom HK, Knip M: Genetic markers, humoral autoimmunity, and prediction of type 1 diabetes in siblings of affected children. Childhood Diabetes in Finland Study Group. Diabetes 2000;49:4858.

-9 Dagdelen S, Hascelik G, Bayraktar M: Simultaneous triple organ specific autoantibody profiling in adult patients with type 1 diabetes mellitus and their first-degree relatives. Int J Clin Pract 2009;63:449-456.

-10 Rosário PWS, Reis JS: Frequency of anti-glutamic acid decarboxylase and anti-thyroperoxidase autoantibodies in siblings of patients with diabetes diagnosed in adult life. Int J Clin Pract 2009;63:517.

11 Pittock SJ, Yoshikawa H, Ahlskog JE, Tisch SH, Benarroch EE, Kryzer TJ, Lennon VA: Glutamic acid decarboxylase autoimmunity with brainstem, extrapyramidal, and spinal cord dysfunction. Mayo Clin Proc 2006;81:1207-1214.

12 Vianello M, Tavolato B, Giometto B: Glutamic acid decarboxylase autoantibodies and neurological disorders. Neurol Sci 2002;23:145-151.

$\$ 13$ Lancaster E, Dalmau J: Neuronal autoantigens - pathogenesis, associated disorders and antibody testing. Nat Rev Neurol 2012;8:380-390.

14 Burton AR, Baquet Z, Eisenbarth GS, Tisch R, Smeyne R, Workman CJ, Vignali DAA: Central nervous system destruction mediated by glutamic acid decarboxylase-specific CD4+ T cells. J Immunol 2010;184:48634870.

15 Brusse E, Maat-Kievit J, Van Swieten J: Diagnosis and management of early- and late-onset cerebellar ataxia. Clin Genet 2006;71:12-24. 
Kuchling et al.: GAD Antibody-Associated Late-Onset Cerebellar Ataxia in Two Female Siblings

Table 1. Clinical, laboratory and imaging characteristics of both siblings

\begin{tabular}{llll}
\hline & & Patient 1 & Patient 2 \\
\hline Sex & & F & F \\
\hline Symptoms & Type of onset & Subacute & Subacute \\
& Gait ataxia & + & + \\
& Nystagmus & + & + \\
& Vertigo & + & + \\
& Dysarthria & - & + \\
& Vertical diplopia & + & 76 \\
Age & At study & 74 & 70 \\
& At disease onset & 73 & 69 \\
\hline At diabetes onset & 74 & normal \\
& MRI & normal & not conducted \\
\hline Autoimmuging diseases & T1DM & normal & + \\
& Others & + & Grave's disease \\
\hline Laboratory findings & Serum GAD65 IgG (ELISA) & $>2,000$ IU/ml & $>2,000$ IU/ml \\
& CSF GAD65 IgG (ELISA) & + & + \\
& CSF oligoclonal bands & + & + \\
& Other autoantibodies & anti-TPO antibodies & anti-TSI antibodies \\
\hline
\end{tabular}

PET = positron emission tomography; ELISA = Enzyme-linked immunosorbent assay; TPO = thyroid peroxidase; TSI = thyroid-stimulating immunoglobulin. 


\section{Case Reports in Neurology}
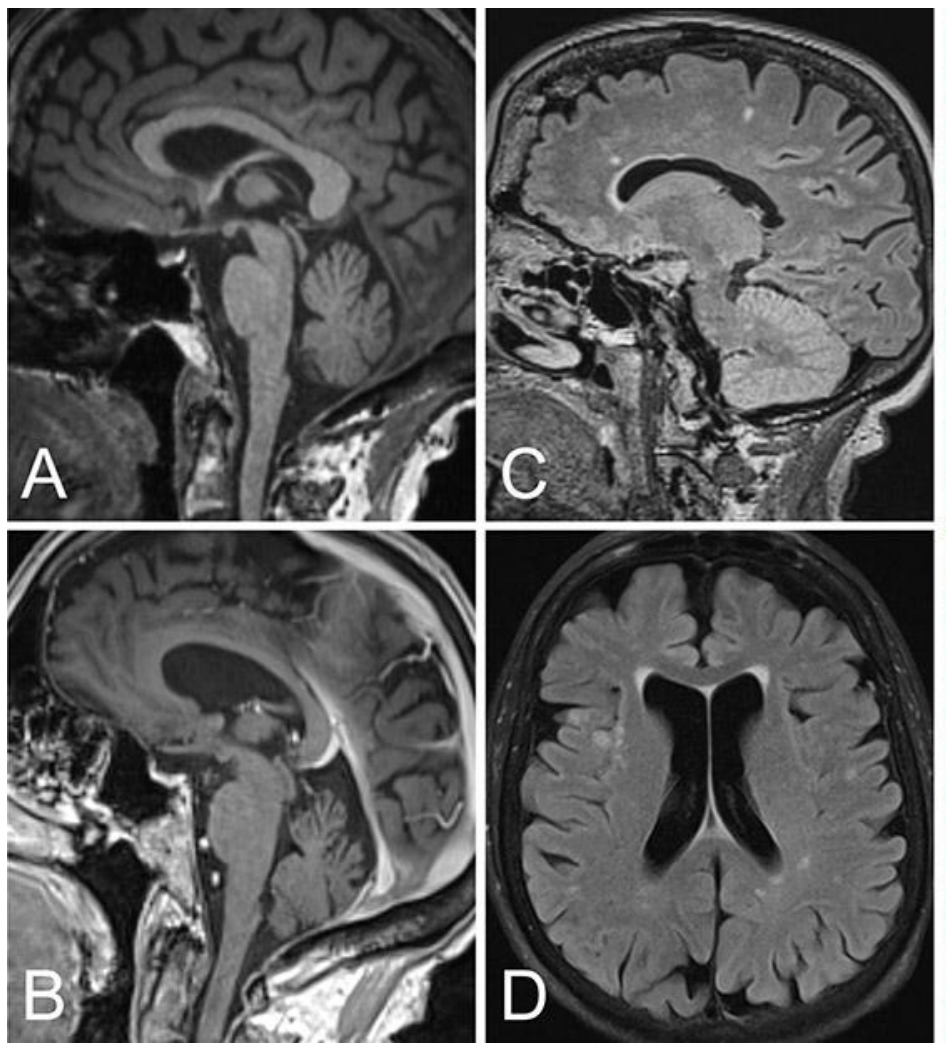
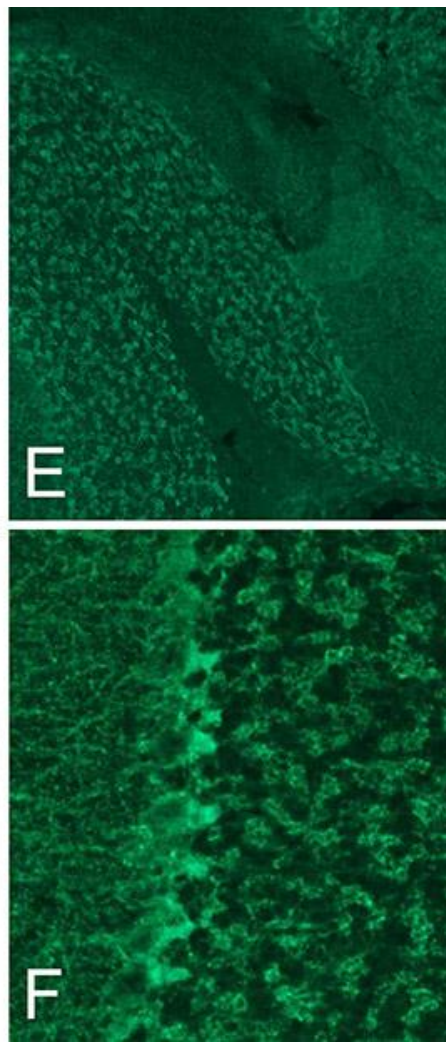

Fig. 1. MRI findings and antibody binding in the cerebellum. MPRAGE (a, b) and FLAIR (c, d) MRIs showing mild vermian atrophy and white matter lesions in patient $1(\mathbf{a}, \mathbf{c})$ and patient $2(\mathbf{b}, \mathbf{d})$. The longer disease duration in patient 2 was associated with mild generalized brain atrophy and leukoencephalopathy (likely microangiopathic) (d). Immunohistochemistry on rat brain sections using serum and CSF of both patients shows the typical GAD expression pattern in the cerebellar granule cell layer (e). Higher magnification demonstrates the punctuate localization in GABAergic terminals, in particular around Purkinje cells (f). 